\title{
Low-Power Wide-Area technologies as building block for smart sensors in air quality measurements
}

\author{
M. Knoll, P. Breitegger, A. Bergmann
}

At present, air pollution monitoring is carried out at low spatial resolution due to high costs, coming along with high accurate measurement equipment. Therefore, to expand the air pollution measurement density, data is fed into dispersion models, which only provide approximate results. To overcome this issue, a much denser sensor network is required, which is directly able to monitor air pollution values.

With the emerging technologies in the Wireless Sensor Network (WSN) area, extensive analysis of air pollution can be achieved. Especially Low-Power Wide-Area Networks (LPWAN) such as LoRa, Sigfox or NB-loT enable smart sensing of wide areas with low power consumption.

After introducing why air pollution measurements are indispensable and which pollutants are measured, it is discussed how LPWAN enable dense spatial resolution. Subsequently, the LPWAN technologies LoRa, Sigfox or NB-IOT are introduced and compared. This is followed by distance measurements and a path loss evaluation in the urban area of Graz using LoRa, where we reached communication distances of up to $1.740 \mathrm{~m}$

Keywords: air pollution measurement; smart sensor; LPWAN; WSN; LoRa

\section{Sensornetzwerk-Technologien als Grundlage für smarte Sensoren bei Messungen der Luftverschmutzung.}

Derzeit werden Luftgütemessungen mit Hilfe von teuren, sehr genauen Messgeräten mit nur niedriger räumlicher Auflösung durchgeführt. Um die Dichte der Messwerte zu erweitern, werden Ausbreitungsmodelle verwendet, welche aber nur Resultate mit großer Schwankungsbreite liefern. Um dieses Problem zu lösen, wird ein dichteres Sensor-Netzwerk benötigt, welches direkt in der Lage ist, die Luftverschmutzung zu messen.

Mit den aufkommenden Technologien im Bereich der kabellosen Sensor-Netzwerke (engl. Wireless Sensor Network) werden großflächige Analysen der Luftverschmutzung ermöglicht. Speziell Sensornetzwerk-Technologien mit geringem Stromverbrauch (engl. Low Power Wide Area Network, LPWAN), wie zum Beispiel LoRa, Sigfox oder NB-loT, ermöglichen eine hohe räumliche Auflösung der Sensorik.

In dieser Arbeit wird zuerst beschrieben, warum Luftgütemessungen unverzichtbar sind und welche Luftbestandteile in der Praxis gemessen werden. Anschließend wird diskutiert, wie LPWAN-Technologien hohe räumliche Auflösung ermöglichen. Weiteres werden die LPWAN-Technologien LoRa, Sigfox und NB-IOT kurz beschrieben und miteinander verglichen. Danach werden Distanzmessungen mit der LoRa-Technologie in Graz vorgestellt, bei denen Kommunikationsreichweiten bis zu $1.740 \mathrm{~m}$ erreicht wurden.

Schlüsselwörter: Luftgütemessungen; smarte Sensoren; LPWAN; WSN; LoRa

\subsection{Current status of air pollution monitoring}

\section{Introduction}

Air pollution is adversely affecting our environment and is known to be a huge health risk when specific limits are exceeded (cf. [1]). Pollutants are emitted from different sources, including human related (anthropogenic) and natural sources. Anthropogenic sources are mainly fuel combustion, transport, domestic heating, industry and agriculture. Currently, in the EU, twelve pollutants are measured in urban areas. Of utmost importance are the mass concentration of particulate matter (PM), of diameter smaller than $2.5 \mu \mathrm{m}\left(\mathrm{PM}_{2.5}\right)$ and smaller than $10 \mu \mathrm{m}\left(\mathrm{PM}_{10}\right)$ and the gases ozone $\left(\mathrm{O}_{3}\right)$, nitrogen dioxide $\left(\mathrm{NO}_{2}\right)$, sulfur dioxide $\left(\mathrm{SO}_{2}\right)$ and carbon monoxide (CO), since the permitted exceedances each year are enforced by the EU Directive 2008/50/EU [2]. The corresponding short term and long term limits are given in Table 1
Currently, air pollution monitoring is done at coarse spatial resolution. This is mainly because of the high costs which come along with high accurate measurement equipment and missing network technologies. In Graz and Vienna, the two biggest cities in Austria, air pollution monitoring is done with 7 and 17 measurement stations, respectively. In Vienna for example, only one measurement station per 100.000 inhabitants exists. Additionally, in many stations, not all types of pollutants are measured. Therefore, to enable air pollution

Knoll, Markus, Institute of Electronic Sensor Systems, Graz University of Technology, Inffeldgasse 10/I, 8010 Graz, Austria (E-mail: m.knoll@student.tugraz.at); Breitegger, Philipp, Institute of Electronic Sensor Systems, Graz University of Technology, Inffeldgasse 10/II, 8010 Graz, Austria; Bergmann, Alexander, Institute of Electronic Sensor Systems, Graz University of Technology, Inffeldgasse 10/Il, $8010 \mathrm{Graz}$, Austria 
Table 1. Air pollution limits of the most common measured pollutants specified by the EU Ambient Air Quality Directives

\begin{tabular}{|c|c|c|}
\hline Pollutant & Short term limit & Long term limit \\
\hline $\mathrm{PM}_{10}$ & $50 \mu \mathrm{g} / \mathrm{m}^{3}$ (1 day) & $\begin{array}{l}40 \mu \mathrm{g} / \mathrm{m}^{3} \text { (calendar year) } \\
\text { Should not be exceeded on more than } 35 \text { days/year }\end{array}$ \\
\hline $\mathrm{PM}_{2.5}$ & & $25 \mu \mathrm{g} / \mathrm{m}^{3}$ (calendar year) \\
\hline $\mathrm{O}_{3}$ & $\begin{array}{l}180 \mu \mathrm{g} / \mathrm{m}^{3} \text { ( } 1 \text { hour) } \\
120 \mu \mathrm{g} / \mathrm{m}^{3} \text { (daily } 8 \text { hour mean) } \\
\text { Should not be exceeded on more than } 25 \text { days per year } \\
\text { averaged over } 3 \text { years }\end{array}$ & \\
\hline $\mathrm{NO}_{2}$ & $\begin{array}{l}200 \mu \mathrm{g} / \mathrm{m}^{3} \text { ( } 1 \text { hour) } \\
\text { Should not be exceeded on more than } 18 \text { hours/year }\end{array}$ & $40 \mu \mathrm{g} / \mathrm{m}^{3}$ (calendar year) \\
\hline $\mathrm{SO}_{2}$ & $\begin{array}{l}350 \mu \mathrm{g} / \mathrm{m}^{3} \text { ( } 1 \text { hour) } \\
\text { Should not be exceeded on more than } 24 \text { hours/year } \\
125 \mu \mathrm{g} / \mathrm{m}^{3} \text { ( } 1 \text { day) }\end{array}$ & \\
\hline $\mathrm{CO}$ & $10 \mathrm{mg} / \mathrm{m}^{3}$ (daily 8 hour mean) & \\
\hline
\end{tabular}

measurements at much denser spatial resolution, a different concept is required. Different concepts exist for enhancing resolution, which includes WSN - in specific LPWAN. These technologies enable high resolution sensor meshes, but also bring along disadvantages, in particular the utilization of cheap sensors with unacceptable large measurement uncertainties. The focus of this paper will be laid on network technologies. An extensive discussion on low-cost air pollution sensors can be found in [3].

\subsection{What Wireless Sensor Networks (WSN) can provide}

Several projects and publications exist where WSN have been used to enhance spatial resolution in air pollution monitoring. In Zurich, Hasenfratz et al. [4] created a mobile sensor network by mounting sensors on trams and busses. Data collection was implemented using the GSM network. Additionally, ozone sensors have been mounted on bicycles. Using this approach it was possible to cover an area of about $100 \mathrm{~km}^{2}$. The CITI-SENSE project (2012-2016) [5] deployed fixed, mobile and personal nodes in eight cities in Europe. Different sensor principles and solutions for fixed and mobile nodes have been compared. Sensors showed good results in laboratory measurements, but much degraded performance in field tests, where differences in humidity and temperature had strong impacts on the measurement results. They suggested that proper sensor technology development is the requirement for spatial dense sensor networks.

In the USA, the Community Air Sensor Network (CAIRSENSE) project [6] monitored air pollution in a $2 \mathrm{~km}^{2}$ area, using fixed stations and four wireless sensor nodes, which have been supplied by solar power and partly by AC power. Xbee radios have been used for communication, which facilitated a maximum communication range of $500 \mathrm{~m}$ in a suburban area. Two nodes with a distance of about $1.5 \mathrm{~km}$ from the base station were unable to report data via wireless communication, as messages were not received. Furthermore, strong sensor dependency on humidity and temperature has been reported with most sensors providing unreliable results. Among others, these projects show possibilities of enhancing the spatial density, whereas still problems exist in bringing together spatial density with accurate sensor resolution.

\section{Low Power Wide Area Network (LPWAN) technologies}

In the last years several LPWAN technologies like Sigfox, NB-IoT or LoRa have emerged. In contrast to classical WSN technologies such as ZigBee, WLAN or Bluetooth, LPWAN feature wide communication range and low power consumption, which come along with low data rates. Therefore, they cannot be used for video or audio data streaming, but are perfect fits for sensing applications, which do not need to transmit big amounts of data. The low power consumption enables battery powered device operations of up to several years. Further, the number of base stations and cost of WSN can be reduced. In the following, three major LPWAN technologies are described.

\subsection{LoRa}

LoRa, which stands for "long range" is a chirp spread spectrum (CSS) modulation technology [7] from Semtech Corporation. It features on the one hand the modulation scheme and on the other hand a media access control (MAC) protocol called LoRaWAN, which is promoted by the LoRa Alliance ${ }^{\mathrm{TM}}$. LoRa operates in the Industrial, Scientific and Medical (ISM) bands of 433-, 868- or 915-MHz depending on the region where it is used. Depending on the used transceiver, it is able to provide a constant RF output power of $20 \mathrm{dBm}$ and data rates of up to $37.5 \mathrm{kbps}$, with the latter strongly dependent on the chosen parameters. Among others, these parameters are spreading factor (SF), bandwidth (BW) and code rate (CR). They influence data rate, communication distance and its robustness to interference. A higher spreading factor and a lower bandwidth result in higher sensitivity, but in lower data rates. The maximum sensitivity of end nodes is $-146 \mathrm{dBm}$. Several publications [8-10] tested parameter settings and the resulting effects on reliability and communication distance. For instance, Augustin et al. [8] reached communication distances of up to $3.400 \mathrm{~m}$ in a suburban area in Paris.

As mentioned before, LoRaWAN is the MAC protocol developed by the LoRa Alliance ${ }^{\mathrm{TM}}$. The specification defines three different types of end devices, which are listed in the following. The power consumption increases from class $A$ to class $C$. 
Table 2. Comparison of LPWAN technologies

\begin{tabular}{|c|c|c|c|}
\hline Attributes/Technology & LoRa $(S \times 1276)^{1}$ & Sigfox (S2-LPQRE) ${ }^{2}$ & NB-IOT (SARA-N2)2 \\
\hline Frequency & $\begin{array}{l}\text { Unlicensed ISM bands } \\
(433-, 868-, 915-\mathrm{MHz})\end{array}$ & $\begin{array}{l}\text { Unlicensed ISM bands } \\
\text { (868 MHz, 902-920 MHz) }\end{array}$ & $\begin{array}{l}\text { Licensed LTE frequency } \\
(700-, 800,-900 \ldots \mathrm{MHz})\end{array}$ \\
\hline Range & $\begin{array}{l}\text { Up to several km, depending } \\
\text { on parameters }\end{array}$ & Up to several km & Up to several km \\
\hline $\mathrm{RX}$ sensitivity & $-148 \mathrm{dBm}$ & $-130 \mathrm{dBm}$ & $-135 \mathrm{dBm}$ \\
\hline Data rate & $37.5 \mathrm{kbps}$ & $600 \mathrm{bps}$ & DL: 27.2 kbps/UL: 62.5 kbps \\
\hline Maximum payload & 255 bytes & 12 bytes & 1600 bytes \\
\hline \multirow[t]{2}{*}{ Power consumption } & TX: 29 mA (13 dBm) & TX: 20.6 mA (14 dBm) & TX: 100 mA (13 dBm) \\
\hline & $\mathrm{RX}: 12 \mathrm{~mA}$ & $\mathrm{RX}: 8.6 \mathrm{~mA}$ & $\mathrm{RX}: 46 \mathrm{~mA}$ \\
\hline $\begin{array}{l}\text { Price per transceiver } \\
\text { (1000 pcs) }\end{array}$ & $\sim 3,66 €$ & $\sim 1,26 €$ & $\sim 10 €$ (for first available transceiver) \\
\hline Network Topology & $\begin{array}{l}\text { Star topology, end devices do } \\
\text { not have specific gateway }\end{array}$ & $\begin{array}{l}\text { Star topology, end devices do } \\
\text { not have specific gateway }\end{array}$ & $\begin{array}{l}\text { Cellular network, end devices have } \\
\text { specific gateway }\end{array}$ \\
\hline
\end{tabular}

- Class A: Uplink (from end node to the gateway/server) messages can be sent any time. Downlink windows are defined after uplink message.

- Class B: Extension of Class A with additional scheduled windows for downlink messages.

- Class C: End nodes listen always except when transmitting.

Additionally, LoRaWAN features a security stack using the Advanced Encryption Standard (AES) for secure communication and message authentication. LoRa networks can be custom built without paying substantial network fees, but the technology is protected and only the company Semtech is manufacturing LoRa ICs and distributing licenses.

\subsection{Sigfox}

Sigfox is an Ultra-Narrow Band (UNB) technology with binary phase shift keying (BPSK), developed by a company with the same name. In Europe, Sigfox uses the $868 \mathrm{MHz}$ ISM band, while in the rest of the world the band in between 902-920 MHz. Transmission power and power consumption are similar to LoRa, with a maximum RF output of $14 \mathrm{dBm}$. The maximum data rate is specified with $600 \mathrm{bps}$, which is sufficient for most sensor applications. The network structure of Sigfox and LoRa is the same, whereby end nodes are not bound to specific gateways. Therefore, several gateways are able to receive and forward the messages, with a server or backend software responsible for filtering equal messages forwarded by multiple gateways. This approach extends the reliability of the network. The business model differs from that of the LoRa Alliance ${ }^{\mathrm{TM}}$, in which the transceiver technology is provided to silicon manufacturers. In contrast to LoRa, the network and software is sold as a service with maximum number of messages per node and day dependent on subscription level. Both uplink and downlink messages are possible, whereby downlink slots for end devices specified directly after sending message to the gateway. Currently Sigfox is covering 45 countries and an area of 3.8 million $\mathrm{km}^{2}$ with their network.

\subsection{Narrow Band-Internet of Things (NB-loT)}

Narrow-Band Internet of Things (NB-loT) has been developed by the 3rd Generation Partnership Project (3GPP). In contradiction to LoRa and Sigfox it is deployed in authorized spectrum and is employing existing cellular (mobile) networks, which are up to now,

${ }^{1}$ Used transceiver for comparison. used for human-oriented communication. The required bandwidth is $200 \mathrm{kHz}$ and current GSM bands, guard bands of the LTE carrier or unused blocks in the LTE band are frequencies at which NB-loT could be deployed. Uplink and downlink communication use different frequencies, whereas data rates are limited for uplink to $20 \mathrm{kbps}$ and for downlink to $200 \mathrm{kbps}$. NB-loT reuses and enhances the LTE protocol, while reducing the functionalities to a minimum for loT usage. The first NB-IOT transceiver (SARA-N2) which is on the market features a maximum output power of $23 \mathrm{dBm}$ and input sensitivity of $-135 \mathrm{dBm}$, requiring for this setting $220 \mathrm{~mA}$ for transmission. Lauridsen et al. [11] compared the coverage of GPRS, NB-IOT, LoRa and Sigfox in a $7.800 \mathrm{~km}^{2}$ area in Denmark, using radio coverage simulations, resulting in the best outcome for Sigfox and NB-loT, especially for indoor connectivity. Apart from these simulations, real distance measurements using Sigfox or NB-loT are not published until now.

\subsection{Comparison}

In Table 2, LoRa, Sigfox and NB-IoT are compared with the respect to different factors. LoRa and Sigfox are deployed in license free bands in contrary to NB-loT, which is using licensed LTE frequency bands. Communication range cannot be directly specified, as it is very dependent on the environment. However, it is expected that all three technologies are capable of reaching several kilometers, at least in suburban areas. The maximum data rate of Sigfox (600 bps) lags behind the other two competitors, whereas the bit rate of LoRa depends on the selected PHY settings. Comparing similar output power, the SARA-N2 NB-loT transceiver [12] requires $100 \mathrm{~mA}$ for $13 \mathrm{dBm}$ TX power and $46 \mathrm{~mA}$ for RX. This is in case of transmission and listening up to five times the required power consumption of LoRa and Sigfox. Sigfox and LoRa are using star network topologies with nodes not directly linked to a specific gateway in contrast to NB-loT. Mekki et al. [13] compared LPWAN technologies on different factors like range, battery lifetime, scalability or payload length, which showed the similarities between LoRa and Sigfox in contrary to NB-IoT.

One main limitation of LoRa and Sigfox provides the ERC Recommendation 70-03 [14] which limits the duty cycling to 1 percent (exception is one band with 10 percent) and the maximum output to $14 \mathrm{dBm}$ (exception is one band with $27 \mathrm{dBm}$ ) in the unlicensed ISM bands.

\section{Experimental evaluations using LoRa}

The following chapter presents distance measurements using LoRa. Afterwards, the path loss is compared to a theoretical model. Finally 

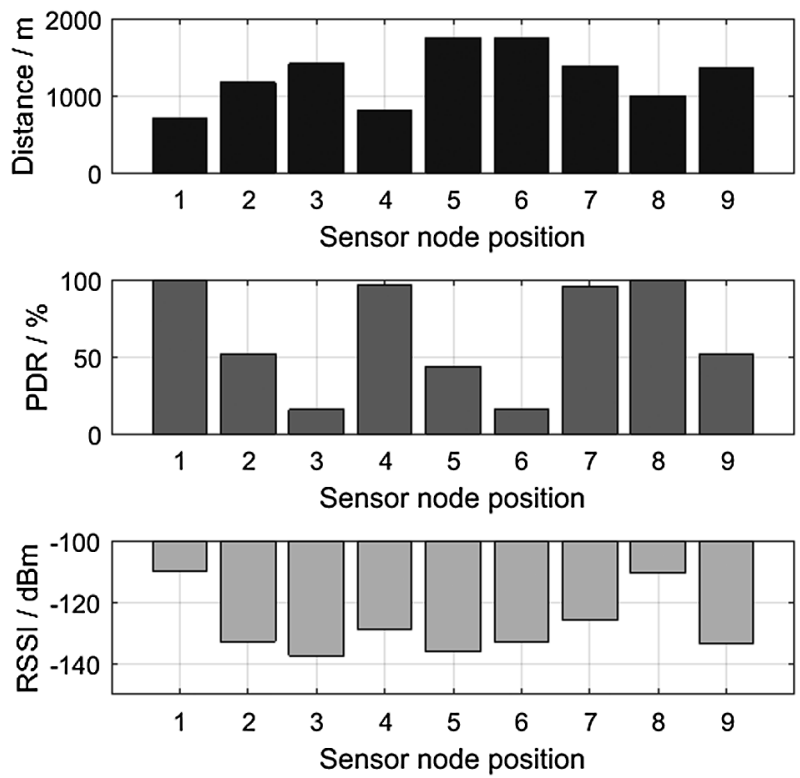

Fig. 1. Results of distance measurements for the different sensor node positions. The top plot shows the distance of the different nodes from the sink. In the second plot the packet delivery ratio from each position can be seen. In the bottom figure the averaged RSSI values from all positions are shown

the potential of the three investigated LPWAN technologies for air pollution monitoring is discussed.

The measurements were carried out in the city of Graz. For these measurements an STM32 Nucleo board with an expansion board, featuring a LoRa SX1272 transceiver, was utilized. The transceiver was used for sending (node) and receiving (sink) messages. The transceiver features a sensitivity of down to $-137 \mathrm{dBm}$. Transmission power was set to a maximum of $14 \mathrm{dBm}$, which results in a maximum link budget of $157 \mathrm{~dB}$, including the onboard power amplifier (PA) and the attached antenna (transmission power in total $20 \mathrm{dBm}$ ). In order to reach the maximum range, the LoRa parameters were set accordingly ( $\mathrm{SF}=12, \mathrm{BW}=125 \mathrm{kHz}, \mathrm{CR}=4 / 8$ ). The payload of each message contained 16 bytes including a message counter for verification. Furthermore was the transmission interval set to 4 seconds and at least 200 messages were sent per node and position. During the measurement cycle the ambient temperature was about $25^{\circ} \mathrm{C}$. In Fig. 2 the sink position, sensor node positions (1-9) and distances between these positions are shown. While the distance measurements were mainly performed in south-east districts of Graz, position 6 was located in the center of Graz. The sink was positioned on the third floor outside the window at the university campus Inffeldgasse. Further were the sensor nodes placed in a typical air pollution sensor height of $1 \mathrm{~m}$.

\subsection{Measurement results}

The measured results are shown in Fig. 1. It was possible to receive messages from all positions. As expected, the packet delivery ratio (PDR), in which only messages without any payload error were counted, is strongly depended on the communication distance. A PDR of more than 95 percent could be achieved from four positions $(1,4,7,8)$, with distances between $710 \mathrm{~m}$ and $1.380 \mathrm{~m}$, while the worst PDR were measured at positions 3 and 6 , with 15 and 16 percent respectively. At position 5 , having a distance of $1.740 \mathrm{~m}$ from the sink, 43 percent of the messages could be properly re-

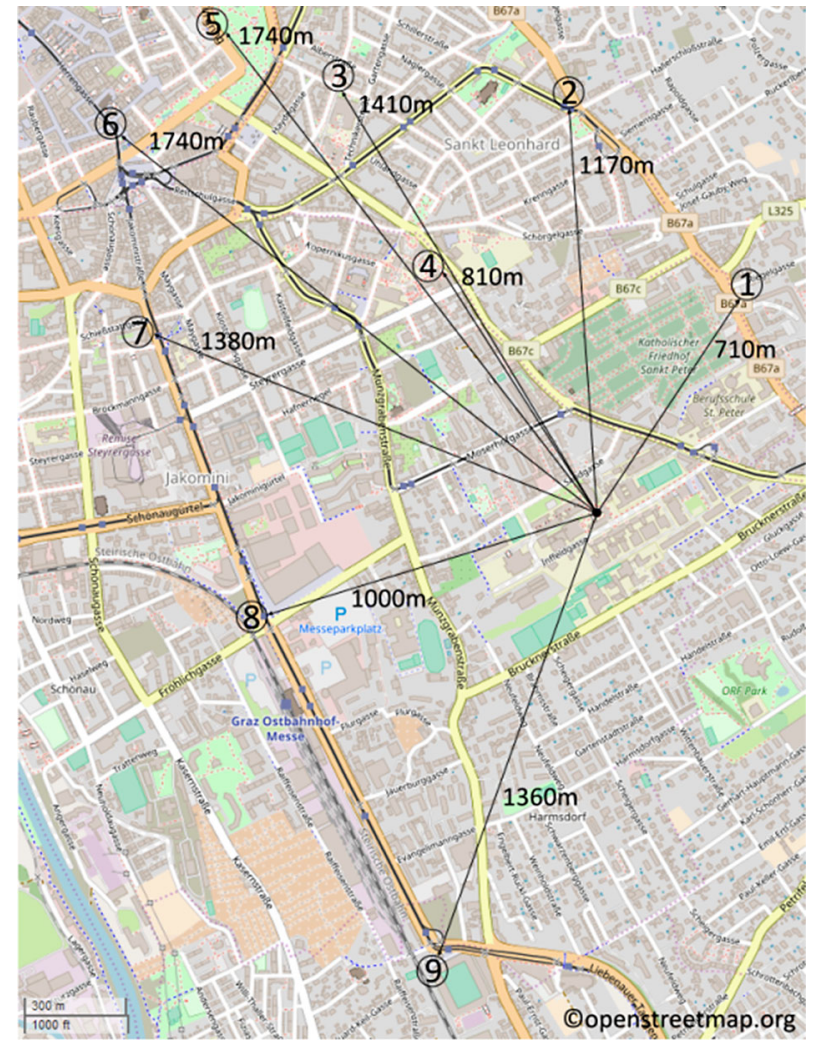

Fig. 2. Map of the distance measurement showing sink, node positions (1-9) and the distance between them

ceived. Generally speaking, the PDR correlates with the node distances, but dependency on topography and the number of buildings in between cannot be neglected.

Furthermore, the averaged RSSI values are shown in the bottom plot of Fig. 1. It becomes apparent, that the RSSI values at position 1 and 8 are significantly higher than from other positions. That can be explained by the descending terrain, which was leading to a close to line of sight (LOS) positioning. In addition to that, results a higher RSSI magnitude in a weaker signal, which indicates a higher distance. This increasing distance was leading to more packets with wrongly delivered payload.

Comparing these results to the measurements from Augustin et al. [8], only smaller distances in a more urban area have been measured. Furthermore, it has to be mentioned, that by using a more sensitive receiver like the $S \times 1276$, or a custom gateway as message sink, better results can be achieved. The reliability of the communication link can additionally be extended, by resending lost messages.

\subsection{Comparison with Okumura-Hata path loss model} In this section the measured path loss is compared to the OkumuraHata Model [15]. This relatively simple model evaluates the path loss for a certain distance between base station and a mobile sensor node. It can be seen as an extension of the free space model. The path loss in $\mathrm{dB}$ is thereby given as:

$$
P L=A+B \cdot \log _{10}(d)+C
$$

Whereby factors $A, B$ are depending on the frequency and the antenna height (of the base station and the mobile node). The distance between the base station and mobile node is specified by $d$ in $\mathrm{km}$. Factors $A$ and $B$ are given using the following formulas:

$$
A=69.55+26.16 \cdot \log _{10}\left(f_{c}\right)-13.82 \cdot \log _{10}\left(h_{b}\right)-a\left(h_{m}\right)
$$




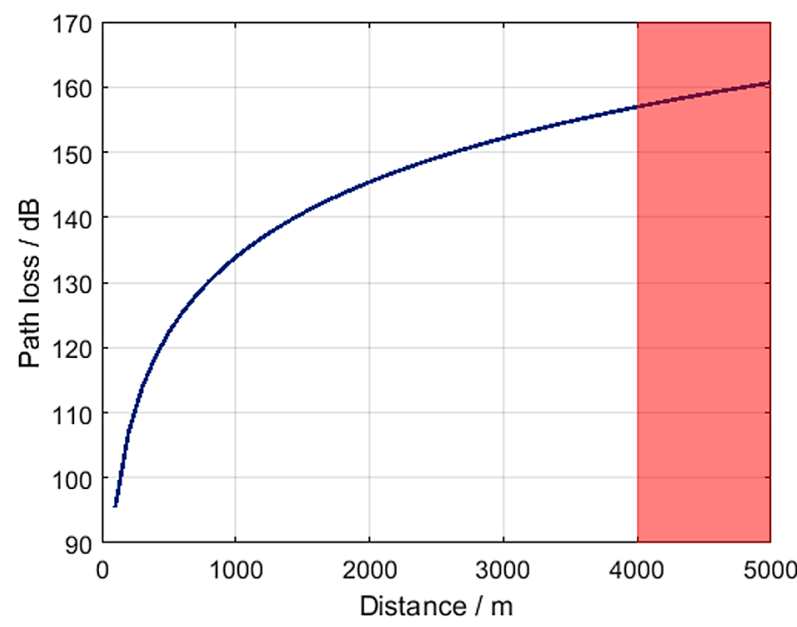

Fig. 3. Okumura-Hata Model for path loss for small and medium sized cities. The red shaded area depicts the distances which are exceeding the total link budget of the used LoRa transceiver

$$
B=44.9-6.55 \cdot \log _{10}\left(h_{b}\right)
$$

With $f_{c}$ represents the used frequency, $h_{b}$ is the base station height and $h_{m}$ the height of the mobile node.

Factors $C$ and $a\left(h_{m}\right)$ are additionally depending on the deployed environment. For small and medium sized cities they can be calculated as followed:

$$
\begin{gathered}
a\left(h_{m}\right)=\left(1.1 \cdot \log _{10}\left(f_{c}\right)-0.7\right) \cdot h_{m}-\left(1.56 \cdot \log _{10}\left(f_{c}\right)-0.8\right) \\
C=0
\end{gathered}
$$

As a result, the evaluated model for distances up to $5.000 \mathrm{~m}$ is depicted in Fig. 3. The red shaded area represents the communication range between base station and mobile nodes, which is not usable due to insufficient link budget in case of the used LoRa SX1272 transceiver. This would result in a maximum communication distance of about $4000 \mathrm{~m}$ in small and medium sized cities. Figure 4 shows the model compared to the measured path loss. The measured path loss scatters due to the terrain and the obstacles in between. This cannot be represented by the simple Okumura-Hata Model, which does not include terrain profile or dominant obstacles. However it can be seen, that the measured path loss values at positions 1, 7 and 8 are well modeled by the Okumura-Hata Model. This is due to the descending area between sink and sensor position, which makes the path loss depending on the distance only. On the other hand, the modeling quality of all other positions is worse. Especially sensor position 3 shows the impact of dominant obstacles. It is directly placed behind the main building of the technical university, resulting in a weak communication link and the highest path loss. It can be concluded, that the OkumuraHata Model is a rough indicator for the communication distance and the density of required base stations. Furthermore needs to be mentioned, that other models exist, which adjust to the area of interest (cf. [16]).

\subsection{Potential utilization for air pollution monitoring}

Benefits of WSNs for low cost air pollution monitoring have already been discussed in Sect. 1.2. The main limiting factors of LPWAN technologies are packet size, data rate and the limitation of maximum transmissions per hour and day. As described in the previous section, the duty cycle and therefore the transmissions per

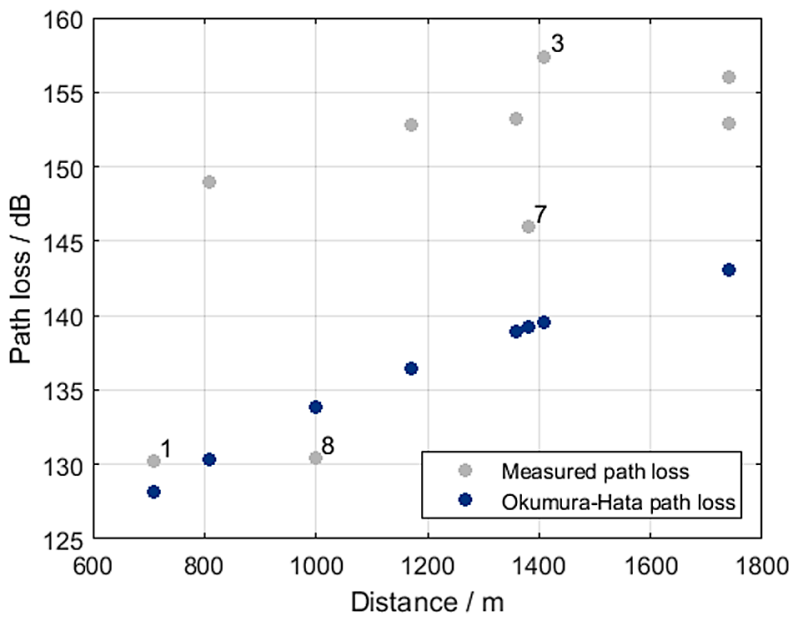

Fig. 4. Comparison of the Okumura-Hata Model for path loss and the measured path loss. Discussed measurement points are labeled

day for the ISM bands, used by Sigfox and LoRa, are limited by the EU. Sigfox additionally confines the number of transmissions by a fee-dependent subscription level. Whether these restrictions are constraining a possible deployment of LPWAN depends on the chosen sensor principle and the data evaluation mechanism. As a consequence, the following factors affect the required data transfer:

- Legislative and technological restrictions are limiting the minimum period at which the measured data can be provided.

- Depending on the sensor principle and the ambient conditions, a frequent remote sensor calibration could be necessary, which increases the required data rate.

- The data processing and evaluation can either be done at the node or at a central processing station, where the latter would significantly increase the transmissions per day.

- Additionally to the pollutant sensor data, date, timestamp and position could also be transmitted.

- If nodes are equipped with additional environmental sensors, such as temperature, humidity or other pollutants, the data rate is increasing.

- The link quality affects the number of transmission as lost or wrongly received packets might be resent.

As the discussion shows, plenty of factors affect the data transfer requirements, which is also shown by Clements et al. [17]. If the principal data evaluation can be done locally, data rate limitations should not confine the utilization of LoRa or NB-IoT. However, the previously discussed restrictions of Sigfox might limit multi-sensor platforms.

Furthermore, additional logic is required at the backend of the network for filtering redundant packets or request wrongly or lost packets. Finally, the evaluation and presentation of the received information should not be underestimated with a vast amount of data to be expected.

\section{Conclusion and outlook}

Overall, it can be concluded that LPWAN technologies offer promising prospects of enabling dense sensor meshes. LoRa and Sigfox bring along the required attributes including long range and small power consumption. NB-loT is the latest technology emerging in this area and future research will show how well the technology will 
perform. Additionally, NB-loT is strongly driven by the mobile communication business, which can be an advantage compared to the other two technologies.

As mentioned in this paper a dense sensor mesh does reduce the spatial resolution, but proper sensor technology has to be used for monitoring air pollution. Otherwise the vast amount of data is redundant when specific limits cannot be reached.

Using LoRa, Graz could be covered using four to six gateways, where possibly hundreds to thousands of sensors are delivering measurement results. Still, a lot of work needs to be done, to bring sensor technologies and network capability together and form proper solutions. Next steps include the development of sensors for the criteria pollutants $\left(\mathrm{NO}_{2}, \mathrm{PM}\right.$ and Ozone), which are a.) miniaturizable, b.) energy efficient and c.) accurate enough to create useful information.

\section{Acknowledgements}

Open access funding provided by Graz University of Technology.

Open Access This article is distributed under the terms of the Creative Commons Attribution 4.0 International License (http://creativecommons.org/ licenses/by/4.0/), which permits unrestricted use, distribution, and reproduction in any medium, provided you give appropriate credit to the original author(s) and the source, provide a link to the Creative Commons license, and indicate if changes were made.

\section{References}

1. WHO (2000): World Health Organization, Regional Office for Europe. Available. http://www.euro.who.int/_data/assets/pdf_file/0005/74732/E71922.pdf [Accessed 26.04.2018].

2. European-Environment-Agency (2017): Air quality in Europe-2017 report. Available. https://www.eea.europa.eu/publications/air-quality-in-europe-2017 [Accessed 20.04.2017].

3. Castell, N., Dauge, F., Schneider, P., Vogt, M., Lerner, U., Fishbain, B., Broday, D., Bartonova, A. (2017): Can commercial low-cost sensor platforms contribute to air quality monitoring and exposure estimates? Environ. Int., 99, 239-302. https://doi.org/10.1016/i.envint.2016.12.007.

4. Hasenfratz, D. (2015): Enabling large-scale urban air quality monitoring with mobile sensor nodes. Dissertation. Zürich: ETH Zürich.

\section{Authors}

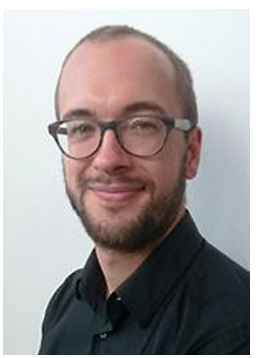

\section{Markus Knoll}

received his B.Sc. degree in Telematics (now renamed to Information and Computer Engineering) from Graz University of Technology, Austria. He is currently finishing his Masters in Information and Computer Engineering at Graz University of Technology writing his master thesis about the development of a low-cost, wireless, photoacoustic based $\mathrm{NO}_{2}$ recognition sensor for air pollution measurements. Since 2014, besides studying, he has been working at NXP Semiconductors Austria $\mathrm{GmbH}$ as a student employee in the verification and validation group.
5. Broday, D.M., Citi-Sense-Project-Collaborators (2017): Wireless distributed environmental sensor networks for air pollution measurement-the promise and the current reality. Sensors, 17(10), 2263. https://doi.org/10.3390/s17102263.

6. Jiao, W., Hagler, G., Williams, R., Sharpe, R., Brown, R., Garver, D., Judge, R., Caudill, M., Rickard, J., Davis, M., Weinstock, L., Zimmer-Dauphinee, S., Buckley, K. (2016): Community air sensor network (CAIRSENSE) project: evaluation of lowcost sensor performance in a suburban environment in the southeastern United States. Copernicus Publications, Atoms. Meas. Tech., 9, 5281-5292. https://doi.org/ 10.5194/amt-9-5281-2016

7. Semtech (2015): LoRa modulation basics. [Online]. Available: 2018. https://www. semtech.com/uploads/documents/an1200.22.pdf [Accessed: 24.04.2018].

8. Augustin, A., Yi, J., Clausen, T., Townsley, W. (2016): A study of LoRa: long range \& low power networks for the Internet of things. Sensors, 17(16), 1466. https://doi.org/10.3390/s16091466.

9. Cattani, M., Boano, C. A., Römer, K. (2017): An experimental evaluation of the reliability of LoRa long-range low-power wireless communication. J. Sens. Actuators Netw., 6(2), 7. https://doi.org/10.3390/jsan6020007.

10. Centenaro, M., Vangelista, L., Zanella, A., Zorzi, M. (2016): Long-range communications in unlicensed bands: the rising stars in the IOT and smart city scenarios. IEEE Wirel. Commun., 23(5), 60-67. https://doi.org/10.1109/MWC.2016.7721743.

11. Lauridsen, M., Nguyen, H., Vejlgaard, B., Kovacs, I. Z., Preben, P., Sorensen, M. (2017): Coverage comparison of GPRS, NB-IoT, LoRa, and SigFox in a $7800 \mathrm{~km}^{2}$ area. In VTC spring, New York: IEEE Press. https://doi.org/10.1109/VTCSpring.2017.8108182.

12. u-blox (2018): u-blox SARA-N2 datasheet. [Online] Available: https://www.ublox.com/sites/default/files/SARA-N2_DataSheet_\%28UBX-15025564\%29.pdf [Accessed: 25.04 .2018$]$

13. Mekki, K., Bajic, E., Chaxel, F., Meyer, F. (2017): A comparative study of LPWAN technologies for large-scale IOT deployment. ICT Express. https://doi.org/10.1016/ j.icte.2017.12.005.

14. Electronic Communications Committee (ECC), (2017): ERC recommendation 70-03. [Online] Available: https://www.ecodocdb.dk/download/25c41779-cd6e/ Rec7003\%20May2018.pdf.

15. Molisch, A. F., (2011). Wirless communications, 2nd edn. Appendix, Chapter 7. [Online] Available: https://www.wiley.com/legacy/wileychi/molisch/supp2/ appendices/c07_Appendices.pdf.

16. Jörke, et al. (2017): Urban channel models for smart city loT-networks based on empirical measurements of LoRa-links at 433 and $868 \mathrm{MHz}$. In IEEE 28th annual international symposium on personal, indoor, and mobile radio communications, PMIRC. https://doi.org/10.1109/PIMRC.2017.8292708.

17. Clements, et al. (2017): Low-cost air quality monitoring tools: from research to practice (a workshop summary). Sensors, 17(11) 2478 https://doi.org/10.3390/ s17112478.

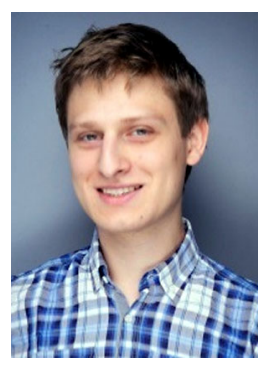

\section{Philipp Breitegger}

received his Master's degree in Technical Physics from Graz University of Technology, Austria, in 2015. He is currently pursuing his Doctoral programme in Engineering Sciences, where he is researching on high spatial resolution sensor systems for monitoring air pollutants in metropolitan areas. In 2016 he worked in a research group with focus on exhaust measurements at AVL List GmbH, before joining the Institute of Electronic Sensor Systems at Graz University of Technology as a university assistant. Since 2018 he has been chapter chair of the IEEE Austria Instrumentation and Measurement Society. 


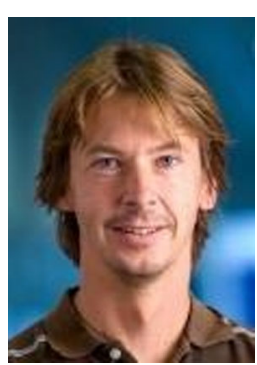

Alexander Bergmann

is head of the Institute of Electronic Sensor Systems at Graz University of Technology (TU Graz), Austria. He received his Dr. rer. nat. from the University of Graz in 2000 Afterwards he worked as assistant and assistant professor at the University of Graz. From 2000 to 2002 he was process $R \& D$ engineer at Infineon Technologies in Villach, Austria. In 2005 he joined AVL List $\mathrm{GmbH}$ and was working as project manager, head of development, head of research and principal scientist in the field of sensor systems. In 2016 he became a full professor at the TU Graz and founded the Institute of Electronic Sensor Systems. His research is dedicated to sensor effects, materials and smart systems for detection of gases as well as aerosols (air polluters and climate forcers) with emphasis on high temporal and spatial resolution. He has published more than 50 peer reviewed publications and 3 book chapters and fielded 25 granted patents. 